\title{
Performance Improvement of OFDM Using Subcarrier Frequency Diversity
}

\author{
Kiran V. Shanbhag \\ (Department of ECE, AITM, Bhatkal,India)
}

\begin{abstract}
Orthogonal Frequency Division Multiplexing is a key contender for broadcasting standards, due to its immunity towards multipath fading and ease of implementation. Here a novel scheme to exploit the frequency diversity within OFDM subcarriers has been proposed which further increases the bit error rate performance. The diversity is achieved by circularly shifting the OFDM subcarrier coefficients in pseudorandom manner. Thus we notice a significant performance improvement without any additional bandwidth requirements.
\end{abstract}

Keywords: OFDM, BER performance, Matlab, Frequency Diversity, Pseudo Noise sequence

\section{Introduction}

Orthogonal frequency-division multiplexing (OFDM) uses a large number of closely spaced orthogonal sub-carrier signals to carry data. The orthogonality allows for efficient modulator and demodulator implementation using the fast Fourier transforms (FFT) algorithm on the receiver side, and inverse FFT on the sender side. The primary advantage of OFDM over single-carrier schemes is its ability to cope with severe channel conditions without complex equalization filters by converting a frequency-selective channel into a parallel collection of frequency flat sub channels. Hence OFDM has developed into a popular scheme for wideband digital communication [1]. Here an effort is made to further improve the performance by utilizing the frequency diversity across the OFDM subcarriers.

\section{Proposed Scheme}

Though several schemes have been proposed to improve the performance of OFDM, very few have tried to exploit the diversity among the subcarriers [2]. In this paper one such scheme has been proposed where the frequency diversity is achieved by using a subcarrier mapper which pseudo randomly distributes a user's subcarriers across the band. Such a distribution offers frequency diversity since the subcarriers would experience diverse fades. Frequency diversity afforded by distributed subcarriers is well suited for users experiencing slowly time varying channels because mobile user experiences different multipath fadings at different time instants, if subcarriers are suitably mapped. The Fig.1 illustrates the subcarriers experiencing different fades in a non-uniform fading channel, at different time instants due to the circular shift by factor $\mathrm{S}$. As we can see at time instant $t_{1}$ subcarrier $\mathrm{X}_{1}$ experiences deep fade but at the same time $\mathrm{X}_{\mathrm{N}-1}$ isn't. Now at instant $t_{2}$, assuming a relatively slowly varying channel, subcarrier $X_{1}$ now experiences no fade but $X_{N-1}$ does, thus can utilize the diversity to minimize effect of fading on particular set of subcarriers.

What makes the scheme attractive is the way the subcarrier mapping is carried out to achieve diversity. This is done by meansof a circular shifter which shifts the IFFT coefficients that are generated at any instant $t$, by a shift factor $S_{k}$, which is governed by a simple PN sequence generator. The shifting of the coefficients is equivalent to the subcarrier shifting.
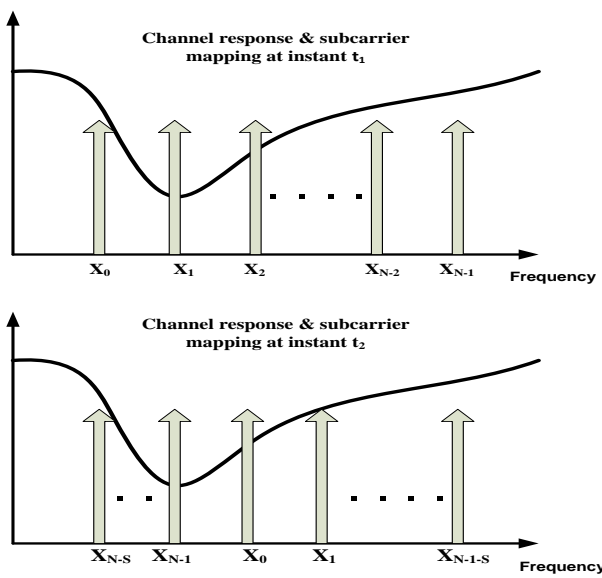

Fig. 1.Illustration of channel response and its effect on subcarrier mapping. As we can see at time instant $t_{1}$ subcarrier $\mathrm{X}_{1}$ experiences deep fade but $\mathrm{X}_{\mathrm{N}-1}$ does not. At $\mathrm{t}_{2}$, it's vice versa 


\section{Implementation}

The figure below shows the complete OFDM implementation employing the proposed scheme. The input data is modulated using suitable modulating scheme. Here BPSK scheme has been used. This modulated data is then passed through a serial to parallel converter, resulting in a set of N parallel BPSK modulated symbols. Then an inverse DFT on these $\mathrm{N}$ symbols is performed using the IFFT algorithm. Here the frequency diversity is achieved by performing $S_{k}$ point circular shift on the set of N IFFT coefficients received for each packet of data where the shift factor $\mathrm{S}_{\mathrm{k}}$ is generated by a simple pseudo noise $(\mathrm{PN})$ sequence generator periodically. The period of the sequence generator is chosen to be equal to or greater than the size of FFT chosen for the OFDM scheme so as to utilize the entire frequency band. As the shift is circular or modular in nature, a shift value greater than $\mathrm{N}$ will not affect the performance. Fig. 2 and Fig. 3 show the transmitter and the receiver implementation blocks. Fig.4 shows the detailed description of the coefficient shifter.

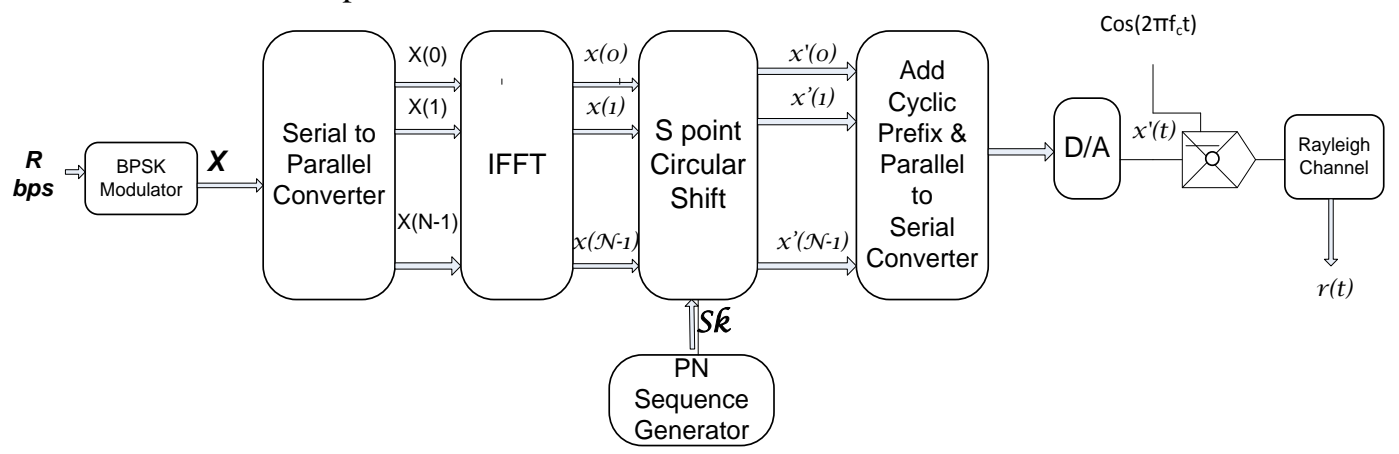

Fig.2 Scheme showing OFDM transmitter with subcarrier diversity

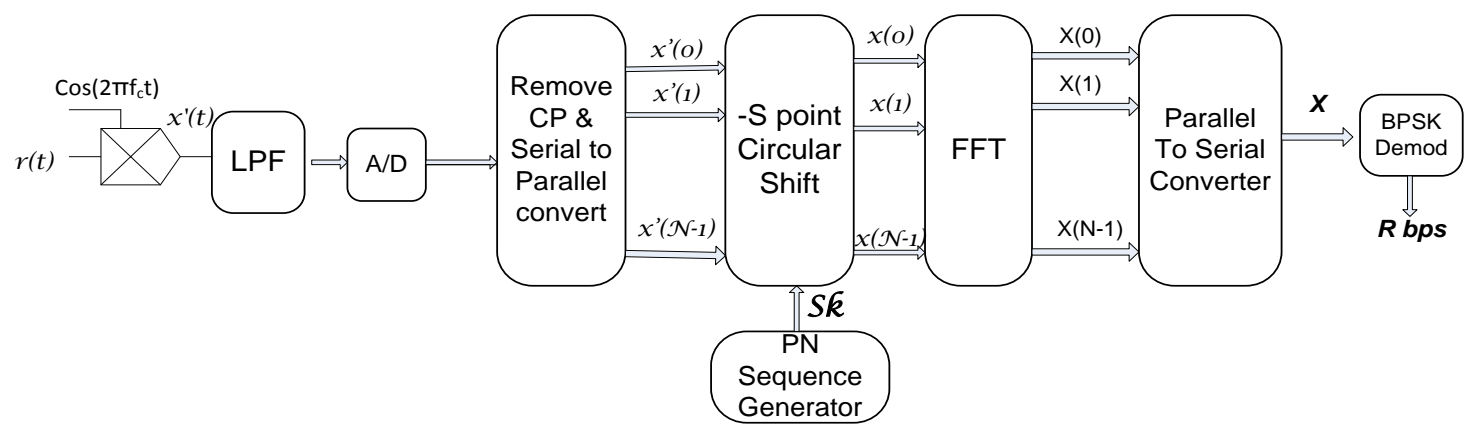

Fig.3 Scheme showing OFDM receiver with reverse shift

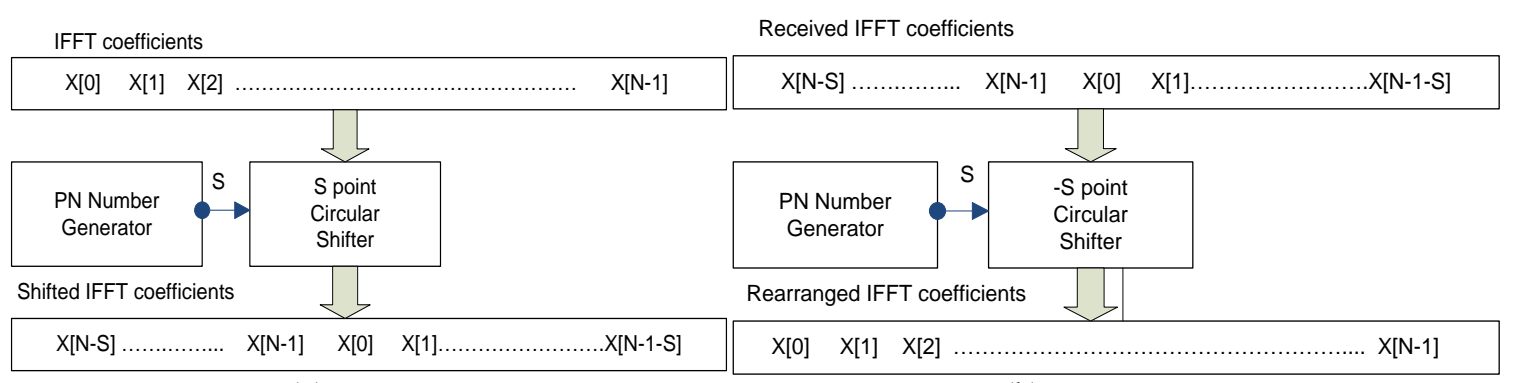

(a)

(b)

Fig.4 The detailed description of coefficient shift scheme

(a) Scheme showing the S point IFFT Co-efficient shift

(b) Scheme showing rearrangement of coefficients by $-\mathrm{S}$ point shift

Fig.4a shows the scheme for scrambling the data by circularly shifting the individual $\mathrm{N}$ IFFT coefficients by $\mathrm{S}$ points, where the value of $\mathrm{S}$ is generated by a PN sequence generator.Fig.4b Shows the rearrangement of IFFT coefficients by reverse shifting the coefficients using the same PN sequence generator at decoder to recover the data.

\section{Simulation Results}

The proposed system was tested on a sample data on Matlab platform and the results have been summarized in this section. BPSK modulation with raised cosine pulse shaping was used. 1024 point IFFT 
\&FFT were used with 16 point cyclic prefix. A PN sequence generator was used to generate a random integer in the range from 1 to 1024. A frequency selective Rayleigh faded channel with AWGN is considered for the simulation. A bit error rate (BER) performance curve was plotted in comparison with conventional OFDM scheme without using the subcarrier diversity. The results have been encouraging as a relative BER performance improvement was observed in the system, as shown in the Figs. 5 \& 6.

Fig. 5 is the simulation result got for a Rayleigh channel with maximum Doppler shift, fd=0.05, with 4 delay paths. We can clearly observe performance gain at higher SNRs as the BER curve deviates from normal OFDM and decays quickly. The system performs similar to conventional OFDM when the fading effect is very low, which is apparent in Fig. 6 where fd=0.001 is employed and no performance difference was found. So it can be said that as the fading increases, the said system performs relatively better.

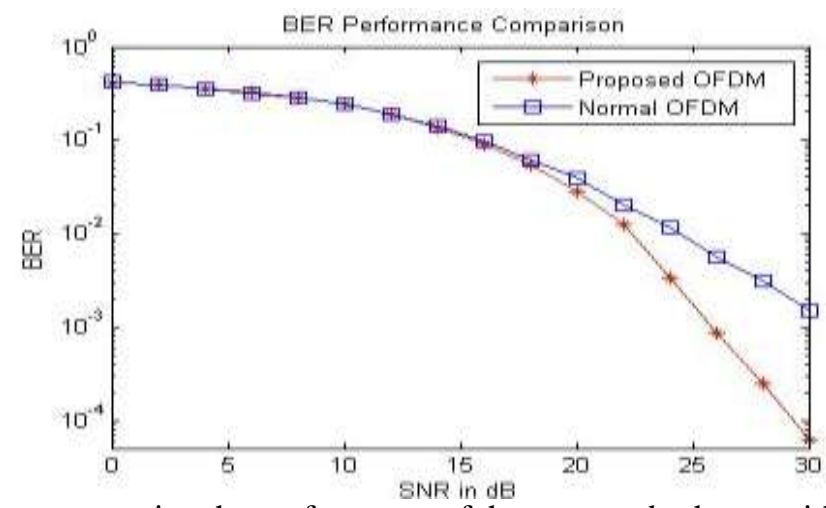

Fig.5 BER vs SNR curves comparing the performance of the proposed scheme with a conventional OFDM scheme without employing the diversity.

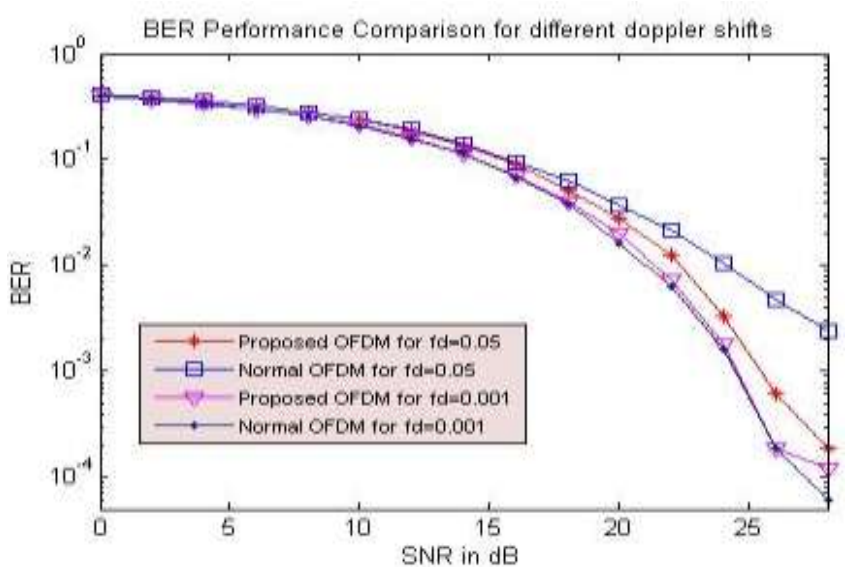

Fig.6 BER performance comparison at two different Doppler shifts, 'fd'. Note the similar performance at lower Doppler shifts but improvement is found for higher shifts.

\section{Conclusion}

A novel scheme to employ frequency diversity among the OFDM subcarriers was proposed by circularly shifting the IFFT coefficients. The scheme used the existing OFDM architecture with a circular coefficient shift block which shifts coefficients in a known pseudo random manner thus exploiting frequency diversity. BER Performance of the system was analysed and compared with conventional scheme and improvements were observed. The fairly simple architecture along with no additional bandwidth requirements makes the system more attractive.

\section{References}

[1] A. Goldsmith, Wireless Communications, Cambridge University Press, Cambridge, UK, 2006

[2] Samuel C. Yang, OFDMA System Design and Analysis, Artech House Library,Norwood, 2010

[3] Wang, T. (R.), J. G. Proakis, and E. Masry, "Performance Degradation of OFDM Systems Due to Doppler spreading," IEEE Trans. on Wireless Communications, Vol. 5, No. 6, 2006, pp. 1422-1432.

[4] J. G. Proakis, D. G. Manolakis: Digital Signal Processing: Principles, Algorithms, and Applications, Prentice. Hall, 2007, 4thedition 